RUNNING HEAD: PROFESSIONALS' VIEWS ON THE NEW SEND

FRAMEWORK

Professionals' Views on New Policy for Special Educational Needs in England: Ideology versus Implementation

Olympia Palikara, Susana Castro, Carolina Gaona, Vasiliki Eirinaki

School of Education, University of Roehampton, London, United Kingdom.

Address for correspondence:

Dr Olympia Palikara

School of Education, University of Roehampton

Froebel College, Roehampton Lane

SW15 5PJ, London, UK

Email: Olympia.Palikara@ roehampton.ac.uk

Tel:+442083928166 


\title{
Professionals' views on the new policy for Special Educational Needs in England: ideology versus implementation
}

\begin{abstract}
Professionals are in the forefront of implementing the new special educational needs and disability (SEND) framework that was introduced in England in 2014. These reforms are expected to raise challenges and opportunities for the professionals responsible for catering for the needs of children with SEND. However, to date, little systematic evidence exists concerning how different groups of professionals perceive these changes. To address this knowledge gap, the views of 349 professionals were ascertained using a semi-structured online survey. The results revealed that although many professionals agreed in principle with some of the key changes introduced, several constrains were reported including tight timelines, budget cuts, difficulties in the collaboration between education, health and care, which suggest a rather fragmented implementation of the new policy. No significant differences were found between groups of professionals for most questions, except for between educational psychologists and special needs co-ordinators on the extension of the provision age range; how well the children's needs are described in the education, health and care plans, and the professional requirements of this process. The findings are discussed in the light of the implications they have for meeting the needs of children with SEND in the face of the fragmented implementation.
\end{abstract}

Key words: professionals, special educational needs framework, England, implementation, SEND

This work was supported by a Froebel Trust Grant 
PROFESSIONALS' VIEWS ON NEW SEND FRAMEWORK

\section{Professionals' views on new policy for Special Educational Needs in England: ideology versus implementation}

\section{Introduction}

The Children and Families Act 2014 introduced a series of changes in the process of assessment and identification of children in need of special support in England and Wales. This was the first substantial change in special educational needs and disability (SEND) policy in over a decade, and as such, brought along a new series of implications for professional practice and service provision for vulnerable children and young people (Castro \& Palikara 2016). A new Special Educational Needs and Disability Code of Practice was introduced to provide statutory guidance on duties, policies and procedures relating to the abovementioned legislation, extending the age of provision from birth to 25 years of age (DfE \& DoH 2015). These reforms are set to present some challenges and opportunities for the professionals concerned with meeting the needs of these children. To our knowledge, this is the first study which aimed to provide novel data concerning different professional groups in education and their views on the implementation of the new SEND framework in the England.

The new SEND Code of Practice follows a person-centred approach to provision, with a strong emphasis on participation of children, their families, and young people in decisionmaking. By adopting this approach, the voices of children and their families, as well as young people acquire a strong position, and must be considered when planning their assessment and intervention. Furthermore, the introduction of Education Health and Care (EHC) plans to replace previous statements of SEND constitute another indicator of the new policy's shift towards an ecological framework for service delivery. The purpose of an EHC plan is to make special education provision for children and young people with SEND focusing on what they 
themselves want to achieve and what support is needed to do this in educational, health and social care terms. Thus, the development of EHC plans fosters multiagency collaboration to learn and discuss the best ways to meet the needs of the child or young person with an increased focus on child participation and quality of life, and the removal of barriers to learning, under the supervision of Local Authority (LA) staff. This process also entails making joint decisions about resourcing (Castro \& Palikara 2016; DfE \& DoH 2015). The integration of service provision outlined by this policy is aptly aligned with recommendations that call for interdisciplinary approaches to disability to promote full inclusion of children and young people with SEND (Castro \& Palikara 2016; Clarke et al. 2011; Norwich 2016).

Although there are many studies that focus on the views of particular professional groups in relation to their work with children and young people with SEND and collaborative approaches to SEND (Dockrell et al. 2017; Palikara et al. 2007; McKean et al. 2017), only a handful of small-scale studies have explored to date the views of professionals about the changes introduced by the Children and Families Act 2014 and the new SEND Code of Practice in England. Prior to the enactment of the Children and Families Act 2014, Pearson et al. (2015) conducted a survey in 2012 in England that aimed to give voice to special educational needs coordinators (SENCOs) to express their views about the potential implications of the changes yet to be introduced for their role working with children and young people. The majority of the SENCOs agreed that the proposed EHC plans would encourage multiagency collaboration in the spirit of promoting more holistic provision, however they raised the question of how this collaboration would be dealt with in practice. Furthermore, SENCOs foresaw their role becoming more bureaucratic, they expected it to require more paperwork and a curtail of their teaching responsibilities in response to becoming instructional leaders for organisational capacity building at school level. In addition, they expressed the view that the development of EHC plans would require skills and knowledge that goes beyond SENCOs' current capacity 
(Pearson et al. 2015). Lastly, the authors noted SENCOs willingness to lead inclusion in schools, but highlighted that this requires well led leadership stemming from policy, suggesting the government's need to communicate clearly what is the offer of support for schools and families, and from whom.

The views of SENCos were also explored by a recent small-scale study, which reported the perspectives of this group of professionals six months following the implementation of the new SEND policy reforms in England. SENCos pointed out the bureaucratic and statutory nature of many of the tasks that they were involved with in the aftermath of the introduction of the reforms including the writing of the schools' SEND policy (Curran et al. 2017). The majority of the SENCos reported that they received support mainly from their LAs, in relation to the implementation of the reforms. However, the results of the study did not indicate whether SENCos were satisfied with the level of training provided, although some of their comments pointed to the limited clarity of the information given. Additionally, both studies reported above (Pearson et al.2015; Curran et al. 2017) focused solely on the views of SENCos related to various aspects of their roles following the introduction of the SEND reforms. The views of other groups of 16 SEND professionals (from education, health and care agencies) were explored in another recent small-scale study (Hellawell 2017). Here, SEND professionals reported feelings of anxiety and insecurity and increased performance demands on their work roles following the implementation of the new policy and 'not knowing' or being provided with very limited guidance by the actual Code of Practice on how to address dilemmas and challenges arising in their daily practice. However, the small sample of the study does not allow for any conclusions to be drawn on whether different groups of SEND professionals may have different perspectives on aspects of the implementation of the new policy framework. Milbourne et al. (2003) argued that a greater understanding of the difficulties experienced by different groups of professionals in implementing policies in such partnerships is required at 
the outset for these to succeed. They state that the ability to tackle social problems through joined-up collaborations from practitioners in different fields is dependent on 'the capacity of different organisations, with different historic projects, based in different disciplines and training, to ensure that their differences are strengths, not limits to collaboration' (Milbourne et al. 2003, 21). A shared sense of aims and tasks needs to be built to avoid misconceptions, assumptions and ambiguities in the roles adopted between and within agencies and individuals, in order to bring together provision that cements existing gaps in services. In this sense, multiagency collaboration offers the promise of combining professional expertise to increase the depth and breadth of responsiveness to difficulties that children and families face in a holistic manner.

The exploration of the views of different professionals and practitioners with regards to service provision for children and young people with SEND reveals a series of implications for practice. The way practitioners and professionals perceive their own role and the role of others with regards to working in SEND contributes to how they deal with the practicalities of policy implementation - how they manage their tasks, responsibilities, collaboration with other services and agencies, and their accountability for provision. To our knowledge, this is the first larger scale study providing evidence on from a more representative sample of professionals regarding various aspects of the implementation of the new SEND policy in England. Additionally, the study examined whether different groups of professionals have different perceptions on various aspects of the SEND policy because of the nature of their professional role.

\section{Materials and Methods}

\section{Respondents}


The online survey was accessed by 374 participants in England and was completed by 349 respondents (307 females, 32 males). 29\% of the respondents were educational psychologists (EPs $n=90), 7 \%$ speech and language therapists (SLT $n=24), 44 \%$ special educational needs co-ordinators $($ SLT $n=154), 4 \%$ headteachers $(n=13), 3 \%$ school teachers $(n=12)$ and $16 \%$ identified themselves as other (other $n=54)$, and were asked to define their role. Responses included assistant headteacher, advisory/ specialist teacher SEND consultant. The majority of the responders were from London $(51 \%, \mathrm{n}=176)$ and the South East of England $(21 \% \mathrm{n}=72)$, while $7.8 \%$ were from the South West of England $(\mathrm{n}=27)$ and $7.8 \%(\mathrm{n}=27)$ from the North West of England. The majority of the responders were very experienced professionals with either more than twenty years of professional experience (42\%), or between eleven and twenty years of professional experience (35\%). $17 \%$ of the respondents had between five to ten years of professional experience and $5 \%$ between one to four. Less than $1 \%$ of the participants had no more than one year of professional experience. In terms of academic qualifications obtained, $33 \%$ of the respondents had a Masters degree, while 25\% reported that they had a PGCE or other postgraduate diploma. $19 \%$ reported that they had a BA/BSc, $14 \%$ a $\mathrm{PhD}$, while $7 \%$ reported having other qualifications. It should be noted that the majority of the respondents (77\%) confirmed that they had specialist training in SEND, while 24\% reported that they didn't have any specialist training in the area. Regarding the type of training they received concerning the implementation of the new SEND framework, half of them (50\%) reported that they had in service training and 135 reported they were offered training by the professional organisation/ body they belong to. $26 \%$ of the professionals reported that they completed some other training, including training offered by the Department of Education, through attendance of Postgraduate Certificate/National Award. However, $10 \%$ of the professionals reported that they were not offered any training in relation to the implementation of the SEND reforms. When asked how satisfied they were with the level of information/ 
training they were offered $60 \%$ of the professionals reported that they were satisfied, and $15 \%$ very satisfied. $21 \%$ reported that they were dissatisfied with the information/ training provided and $4 \%$ very dissatisfied.

\section{Survey}

The semi-structured survey questionnaire was developed following a review of the literature (Castro \& Palikara 2016) and piloted with a number of professionals (educational psychologists, headteacher, SENCos) to clarify questions, answer options and confirm their appropriateness; hence the construct validity of the instrument. Following the completion of the pilot study, minor modifications were made in some of the questions of the survey and the wording was adapted accordingly (copy of the questionnaire is available from the corresponding author). The survey comprised three sections. Section one concerned participants' demographic characteristics and information about their work experience and professional role. Section two included information on the training that education professional received in relation to the introduction of the SEND reforms. Section three asked professionals to rate their position in relation to some of the main changes introduced by the Children and Families Act 2014 and the SEND Code of Practice and to comment.

\section{Procedure}

A link to the survey was sent to schools and education establishments, educational psychology services, speech and language services and their relevant professional organisations. The completion of the survey took between 15-20 minutes. Three reminders were sent before the survey was closed. Participation was voluntary and responses were anonymized. This study was approved by the Research Ethics Committee at the University of Roehampton, which adheres to the British Psychological Society ethics guidelines. 


\section{Data analysis}

The ratings made by the professionals regarding how they position themselves in face of the various policy changes were analysed through descriptive statistics. The qualitative comments that were added to the ratings by the respondents were analysed using inductive thematic analysis. Quotations have been chosen to highlight the common themes identified by a number of professionals. One way-ANOVA analysis was performed to test differences between professionals groups in regards to how they rated the 5-point Likert scale items assessing their views on various aspects of the new policy; Post Hoc tests Tukey and GH were used to establish the differences between groups, dependent on whether the variables under analysis showed homogenous variance or not. Chi-square analysis were also ran to test the difference between professionals in relation to items rated as categorical variables.

\section{Results}

Professionals' Ratings and Comments Regarding the Implementation of the New SEND

\section{Policy}

Results portraying the ratings by the professionals in relation to the main policy changes are presented first, followed by their qualitative elaboration on these ratings. Here, quotations are used to illustrate the themes that emerged from the inductive content analysis; these are labelled by profession of the respondent and their participant number.

Extension of Age Range for Service Provision (0-25)

Professionals were asked to rate and to justify their position regarding the wider age range (from 0 to 25$)$ defined in the new policy for SEND. The vast majority of professionals $(86 \%)$ reported that they agreed with the extension of provision. When asked to justify their position 
in this respect, professionals highlighted the potential of this extension for bridging the gap to further education and employment for young people with SEND:

'Children do not simply lose their need when they turn 16 or 18, the band of 25 may encourage more special needs children into further education and bridge the gap to the work place’. (P352, Inclusion Co-Ordinator)

Similarly, others acknowledged the risk of exclusion and vulnerability of young people with SEND and their families, and highlighted the potential of the extension of age range to enabling a smoother transition to adulthood.

'People with SEND are often very vulnerable and having a wider age range on the EHCP should theoretically support them through the transition into adulthood'. (P360, $S L T)$.

'Moving the definition into early adulthood should enable a clearer pathway for parents/carers/students to allow a greater transition'. (P355, SENCo)

'Children with SEND and their families have always found the transition to adult services traumatic in my experience and one document (if used well) can ease that transition'. (P338, Specialist autism outreach teacher)

However, $10 \%$ of the professionals reported that they did not agree nor disagree with the introduction of the wider age range, while $5 \%$ of professionals said that they did not agree with the introduction of the wider age range. When asked to justify their view, the reported reasons including the further strain on the current funding.

'Although there has not been the funding to support this, it has effectively diluted the existing poor funding further'. (P228, headteacher)

These responses reflect on the multiple dimensions and implications of policy change and implementation. In this respect, following from the quote presented above, professionals 
seemed to question the underlying implications of such introduction, i.e. funding constraints, rather than the measure of a wider age range on itself.

\section{Assessment of SEND Involving Education, Health and Care}

Respondents were asked to rate their position regarding the intention of having an assessment of needs for children with SEND involving health, education and social care. The vast majority of professionals (92\%) reported that they agreed with this intention, while 3\% reported that they did not agree, and a further $6 \%$ that they did not agree nor disagree with this intention. The professionals' comments elicited from this rating highlighted that in their view statutory assessment for children and young people with SEND still mainly very much involves education:

'As the EHCP is always driven by education so it gives a false impression of the involvement of social care and health'. (P371, SLT)

'I agree but am disappointed that essentially we still end up with education plans!'(P370, advisory teacher for SEND)

'Since the change from statements to EHCPS I have not seen an increase in any involvement from other agencies. Schools are still doing all of the work'. (P364, SENCo)

'We needed all services involved to ensure a holistic approach BUT, in my experience (now in mainstream), education are the only ones involved consistently - neither health nor social care have been involved beyond initial assessment. Only speech and language have input but they are in school and so easier to ensure their involvement'. (P325, SENCo)

Respondents reflected on their experience of joining up with other services to provide a holistic view of the child at assessment and intervention levels, acknowledging the existence of needs 
that have implications beyond the boundaries of education. On this matter, some professionals reported several constraints concerning multi-agency team-work in relation to assessment:

'I believe this is a good goal but the reality of coordinating with all the other professionals is difficult in practice'. (P257, EP)

'It is essential that teams work together and we recognise that all these needs link together - the more that we can recognise the links between emotional, education and personal needs the better we can support the individuals. Again, the changes in documentation do not necessarily ensure that this joint working will materialise with current cuts, limited time availability and staffing issues'. (P310, SLT)

'The idea behind this being a joint assessment between all agencies involved is good as many children with complex needs have health and educational needs rather than one or the other. However this does not always work practically in reality and there is still room for improvement in linking these services'. (P250, SENCo)

These comments once again portray that professionals' reflections and concerns seem to be related to practicalities of front-line implementation of the policy, and not necessarily to the underlying principle of multiagency introduced by the Code of Practice.

\section{Replacement of Statements of SEN with EHCPs}

The introduction of EHC plans to replace previous statements of SEND was another topic explored through the survey. Professionals were asked to rate their position in relation to this change introduced by the new policy. Sixty-seven percent of the participants reported that they agreed with the intention of replacing the statements with EHC plans. Those in agreement with this acknowledged several strengths of the EHC plans, including being more personcentred, holistic and highlighted the importance of parental co-production: 
'They are more person-centred, the plan is very outcome-based and easy to be implemented across a range of client groups and those with differing needs. It gives a good opportunity for involvement of further agencies if identified at the time of transfer.' (P351, SLT)

'Holistic care. Better child protection. Reduced teacher bias, less chance for prevention of access to assessment, less intimidation, bullying and threats by teachers who 'just know' and demand blind trust in place of assessment or threaten and bully to silence.' (P339 SEND professional).

'EHCPS are dynamic active documents with parental co-production and therefore more likely to be relevant and up to date, and useful to providers'. (P261, Education Consultant)

On the other hand, $23 \%$ of the respondents said that they did not agree nor disagree with the replacement of the statements by EHC plans. This position was frequently based on the impression that EHC plans were not different to previous statements, and therefore professionals did not find a strong rationale for their adoption and development. Furthermore, respondents referred to the administrative implications of this change, suggesting their development as time and resource consuming.

'Just a change of format'(P364, SENCO)

'It seems to be just a change of name'(P361, SLT)

'At the end of the day, it is a change of paperwork and administration - what is

important is that what is written on the piece of paper is relevant for the individual, and that those targets / support packages are put into place and upheld by the professionals / councils that are involved with the individual (P310, SLT)

'There seems to be no palpable difference in Statements and EHCPS - certainly not worth the money/time spent in really just a redesign of a form with little impact 
beyond change of paperwork and the ensuing delays due to local authorities having to push through changes very quickly'. (P254, SENCo)

Ten percent of the participants reported that they disagreed with the replacement of statements of SEN with EHC plans. A series of themes emerged among these respondents with regards to the introduction of the EHC plans. One salient identified theme concerned the perceived increase by professionals of the paperwork involved in the development of EHC plans:

'We are drowning in paperwork, with no uniformity of request between different LAs and county councils, marginal involvement from health professionals (dependent on where pupils live and access to services) and parent SENDIASS groups involved in creating protocols and documentation that is not achievable. E.g. EP involvement in more than 1 parent meeting (P287, SENCo).

Another theme from this group of professionals related to concerns in meeting the level criteria or threshold to qualify for an EHC plan:

'I can see they may be more person-centred however, some very vulnerable children in the mainstream system will not meet the new high level of criteria necessary'. (P295, SENCo)

'Many children and young people who do not qualify for EHCPs still need lots of specialist support - including allied health professional involvement. This is not available under the current system'. (P318, SLT)

The long delays experienced in the completion of the new EHC plans was another theme linked to the disagreement of professionals on the replacement of statements of SEND with these newly developed documents:

'Apart from TAC meetings, they are largely the same. They still unfortunately tend to focus on education and given how poorly thought out by the government and poorly supported at a local level (no increase in staff to account for the transfer of statements 
to EHCP for example). The legal limit of 20 weeks is rarely achieved. So, although the aim of the EHCPS was to have a quicker process it has turned into a much longer process. It feels like the same thing i.e. still a statement but with greater delay and higher rates of SEN departments' sickness and absence due to the inevitable overwhelm'. (P108, EP)

Professionals also expressed that they were particularly concerned about the writing of plans by people with little training or knowledge of SEN, and about the discrepancies and lack of consistency among different LAs in the procedures followed and paperwork produced:

'Plans that replace statements are often written with limited and out of date advice and are outsourced, written by people who have very limited knowledge of SEN - these are not better. A well written plan written with up to date and detailed information may be better.' $(P 42, E P)$

'The new documents are long-winded and cumbersome. They are not consistent, with regards to the information contained in them, as they are written by a range of different professionals with very little training. Statements were approximately 8 pages long, very clear and easy to find relevant information quickly. The new EHCPs are between 20-40 pages long and each one is quite different from another in terms of content and information'. (P128, SENCo)

'Not a step forward, just created confusion. All LA developed their own paperwork and some SENCos deal with multiple LA, one I've met is dealing with 17 different types of EHCP paperwork and review paperwork’. (P23, EP)

Professionals were also asked to rate their position on whether the EHC plans describe better the children's and young people's needs when compared to the Statements of SEN. Sixty-three percent of participants reported that they agreed with this position. Potential arguments presented in favour of the above statement concerned 
Nonetheless, $32.14 \%$ of professionals reported disagreement in that EHC plans convey a better picture of the needs of children and young people than those conveyed by statements of SEND. Respondents offered reflections on the style, length and overall content of the documents in their responses.

'I don't think there is any improvement'(P371, SENCo).

'Largely these plans are unworkable and badly written. Health aren't involved adequately and only the education part has any legal force. Badly thought out'(P363, SENCo)

'Although they can be up to 50 pages long and are completely unwieldy as a working document. (P228, head teacher)'

'I think there is still a lot of work to be done about how the final plan is written. I don't really feel at the moment there is any difference in the way needs are described'(P122, EP)

'Though the intention is strong, the EHC plans I have seen so far have been very weak, and little more than a paperwork exercise. Many missed opportunities'.(P92, Consultant SEN)

'I feel the part 2 of the old statement did this very well and the needs are far less clear in the new EHCPs' (P327, SLT)

'They are an improvement, but there is a way to go. Provision for needs is still based on what schools can provide. Services should jointly support these children and their families’. (P232, SENCo)

In the question relating to whether the requirements for professionals were different under the EHCP process than to those under the Statementing process, $51 \%$ of the professionals rated this positively, while $35 \%$ reported that they did not perceive their role differently. When asked whether they felt more able to offer a full picture of children's and young people's strengths 
and needs under the EHCP process when compared to the Statementing process, $53 \%$ of respondents answered positively while $33 \%$ negatively.

\section{Transition Process to the New System for SEND Provision: Challenges}

Professionals were asked to describe their position in relation to the transition to the new system for SEND provision. Fifty-five percent of the participants reported that this transition was smooth, and that adequate training and information was provided, while $42 \%$ of the professionals reported that although the transition was smooth, more information and training should have been provided. Three percent of the participants characterised the transition as difficult with not enough information, guidelines or training being provided. Barriers reported by the professionals concerning the transition process to the new system for SEND provision included the tight timeframes in combination with the limited training offered:

'The government has set targets including timeframes, cut budgets and expect more from professionals. It has not been taken into account that we need time and training to support the transition. Most of us have more than one role in school. The parents are also looking to us for answers and support. More training and funding is needed!' (P293, SENCo)

'I had to research the answers to my questions for myself and also relied heavily on my EP for support at first. Local Authority training was patchy and confusing'. (P235, class teacher)

Another salient emerging theme concerned the increase on the workload and pressure on professionals upon the introduction of the new Code of Practice. Respondents pointed to these experiences of constraint during the early stages of implementation at LA level: 
'Ifeel the authority struggled with the additional workload and did not have time to put together coherent guidelines before they started the implementation. This meant the initial EHCPs were delayed and of poor quality. Subsequent paperwork has been reviewed a few times and different schools are using different versions. It would have been more effective and efficient if more time was spent on the planning to implement stage'. (P260, SEN Unit manager).

'As a LA employee the transition process has been immense leading to a huge amount of additional work under tight time scales'. (P370, Advisory teacher for SEND)

On a similar note, other professionals emphasised the implications for their work of ongoing changes on guidance paperwork:

'Ongoing changes have made it challenging to keep up with what is expected of SENCOs'(P184, SENCo)

'There has not been enough training or guidance available to ensure a smooth transition. Any guidance available and forms prepared have been constantly modified and adapted'. (P128, SENCo)

'The transition has been long with endless change in process. This has caused high levels of stress for all who work in SEN'. (P87, EP)

Lastly, another theme that has emerged regarding the challenges of the transition to the new system was the funding available:

'What has been difficult is not reflected above. There has been nothing like enough government funding to support the transition or the implication of providing services and resources for an older age group. SEN Officers and schools have been horribly overstretched in managing this transition'. (P359, SLT) 


\section{Differences between the views expressed by professional groups}

It was then examined whether different groups of professionals had different perceptions on various aspects of the SEND policy. Differences between professional groups were found in some of the variables analysed in the survey; specifically, there were statistically significant differences between educational psychologists and SENCOs in their ratings regarding widening the range of service provision for children and young people with SEND, from birth to 25 years of age $(F(5,249)=3.516, p=.004)$, with psychologists rating this change less positively $(\mathrm{M}=2.7, \mathrm{SD}=1.2)$ than SENCOs $(\mathrm{M}=2.1, \mathrm{SD}=1.0)$.

These two groups of professionals also differed significantly in relation to the extent to which the EHC describe better the children's and young people's needs when compared to the Statements of SEN $\left[\chi^{2}(2, n=216)=13.167, p=.001\right]$ and in relation to the extent to which their requirements as a professional differed under the EHCP process when compared to those under the Statementing process $\left[\chi^{2}(2, n=216)=11.365, p=.003\right]$; in both cases educational psychologists are more pessimistic than SENCOs.

\section{Discussion}

The overall aim of this study was to address the limited availability of evidence concerning the views of professionals on the implementation of the new SEND framework in England through the completion of a semi-structured online survey. Results show that professionals seemed to agree in principle and to a large extent with many of the changes introduced by the new special educational needs framework, including the extension of provision from 0 to 25 , the intention of having an assessment of needs for children with SEND involving health, education and social care and the replacement of statements of SEN with EHC plans. However, it is worth noting that although these ratings are mostly in favour of the idea behind these policy changes, further qualitative comments given by professionals as clarifications of their ratings provide clear evidence of an existing paradox between perceptions 
of ideology and perceptions of practice implementation. The combination of ratings and comments seems to illustrate that, for example, although professionals fundamentally agree with the idea that assessments should involve the concerted efforts of education, health and social care (in the overall rating), further comments show disappointment that in practice the final plan is still very much solely education focused. This paradox between agreeing with the ideology but disagreeing with its practical implementation is consistent across most of the issues rated and commented by the participants, and provides clear evidence of the gap between political ideology and practical provision that has been previously pointed out in the literature (Castro \& Palikara 2016).

Only a few significant differences were found regarding the views expressed by different groups of professionals, namely between educational psychologists and SENCos. However, the themes that emerged from the analysis of the quotes from both professional groups reported several similar constraints and barriers, including the difficulties in implementing multi-agency work, the amount of additional work required, the pressure felt by professionals and the long delays in the completion of EHC plans and statement transfers in light of tight timeframe instated.

\section{The Gap Between Ideology and Implementation}

As presented above, the views of professionals have shown their agreement in principle with the changes introduced by the new legislation, and have highlighted the existence of a gap between ideology and implementation with regards to the new SEND framework in England. While acknowledging this implementation gap, Eccles (2012) highlights a series of tensions occurring between policy making and implementation, suggesting that implementation led by front-line professionals risks the policy being submerged under bureaucratic inertia and professional vested interests, while at the same time policies whose implementation are 
designed by policy makers risk being unworkable for front-line professionals (Eccles 2012). The recognition that individual children and young people have needs that cut across areas within education, health and social care, and that these needs change over time, constitutes a strong rationale for the development of holistic services to meet needs and enhance their quality of life and participation (DfE \& DoH 2015). In order to address these tensions and to support continuous improvement of policy implementation, the Code of Practice discusses the need to provide professionals with opportunities to feedback on the development and implementation of EHC plans (Norwich \& Eaton 2015). In doing so, special attention should be given to the different governance arrangements of the partners involved in collaborative work (Eccles 2012); understanding differences in profession-specific agendas might improve collaborative policy implementation, and shed some light about the believes and views by which different occupational groups deal with meeting the needs of children and young people with SEND from their field (Lindqvist et al. 2011; McKean et al. 2017; Palikara et al. 2007).

In synchrony with the professionals' comments obtained through this survey, Norwich (2014) highlighted that many of the changes introduced by the SEND reforms are not actually new but rather extensions or integration of existing principles. The major changes concerned the funding allocation, the governance and accountability model introduced. However, these reforms affect the way by which education professionals perceive their roles in a changing SEND system with increasing demands that they feel are imposed to them in a climate of austerity, budget cuts and uncertainty in relation to the variability by which these changes are implemented across different LAs. These findings seem to echo those arguing that professional practices and identities are changing through policy making (Ball 2015; Hellawell 2017).

\section{Challenges in Multi-Agency Work Between Education, Health and Care}


One the themes which emerged from the findings of this study concerned the challenges that multiagency collaboration observed at implementation level, especially in relation to the assessment process following the introduction of the EHC plans and the replacement of statements of SEN. In the spirit of making the new SEND policy more user-led with reduced local and central government involvement and regulation, the Code of Practice gives local authorities and health bodies 'considerable freedom in how they work together to deliver integrated support' to improve children and young people's outcomes (DfE and DoH 2015, 44; Norwich \& Eaton 2015). Although the Code of Practice provides relevant information about inter-service collaboration in some detail (Chapter 3), and what an EHC plan should include, it leaves the how to be decided by the local authority with no indication of any guidelines or recommended practices for interdisciplinary assessment and subsequent development of EHC plans and outcomes (Castro \& Palikara 2016; Norwich \& Eaton 2015).

Furthermore, the Code of Practice states that local authorities must produce a local offer, where all information about the provision available locally across education, health and social care for children and young people who have SEND and their families is published (DfE \& DoH 2015). The rationale for this is to provide comprehensive information about the provision available in a given area, and to make this provision 'more responsive to local needs and aspirations' by involving directly in its development and review children and young people with SEND, their families, and service providers (DfE \& DoH 2015, 60). Therefore, there is an expectation that services and provision, and their publication in the local offer will be coproduced, representing a major opportunity to design services that meet the needs of a specific local area, with their users at the centre (Lamb 2013). This complex task requires the recognition of a shared understanding of roles, aims and goals across disciplines and stakeholders in a specific area in order to work collaboratively for their achievement (Lindqvist et al. 2011, McKean et al. 2017). In spite of the introduction of a Local Offer, participants 
expressed their concern and disappointment about the discrepancies and lack of consistency among different local authorities with regards to the procedures and paperwork involved in the development of EHC plans, and in particular regarding the writing of plans by people with little knowledge of SEND.

Challenges in multiagency collaboration have emerged particularly with regards to the assessment process involved in the education, health and care plans. Professionals' perspectives on statutory assessment are that this process is still very much focused on education mainly, in line with the concern expressed by Norwich (2014), who suggested that 'calling the new plans education, health and care plans is also misleading, as they are basically education plans where health and social care needs are included in so far as they relate to special educational needs. They are not, for example, about health provision unrelated to special educational needs" (Norwich 2014, 416).

Both Broach, Clements, and Read (2016) and Nettleton and Friel (2015) suggest that the timescale of implementation of the new SEND framework seems to have been too rushed. Indeed, following up from the 2011 Green Paper that promised to dramatically change the scene for SEND for the first time in decades in England, the Coalition Government set up a pathfinder initiative that aimed to improve the support available for children and young people with SEND, and to improve parental confidence in the system by improving accountability and enhancing parental control and choice (DfE 2011). The idea was for the lessons learned through this pathfinder route to help the drafting of what later became the Children and Families Act 2014, and to support national implementation of reforms (DfE, 2015). However, the timeline for the pathfinders was extended, and the legislation was enacted before the final evaluation report was published in July 2015, almost a year after of the entry into force of the new SEND Code of Practice. 


\section{Commonalities and Differences in Professionals' Views}

To our knowledge, no previous study has examined specifically the differences between professional groups concerning the changes introduced by the new SEND framework. Our findings suggest that the different professional groups had overall similar views on the changes introduced by the new SEND framework. However, significant differences were identified between the views of educational psychologists and SENCos regarding the extension of provision age range; regarding how well the children's needs are being described in the newly introduced education, health and care plans, which replaced the statements of SEN; and regarding whether the requirements presented to professionals in light of the EHC process differ from the requirements presented to them in the old system. These differences between educational psychologists and SENCos may reflect fundamental changes required in the nature of their professional roles following the introduction of the reforms. For example, the extension of the educational psychologists' role now required to work directly with young people aged up to 25 years has been described as the biggest change in the profession over the last 30 years, adding and entirely new facet to their current professional role (Atkinson et al. 2015). These changes necessarily trigger the need for acquiring new competencies in relation to post-school provision.

\section{Conclusions}

Central to the implementation of the new SEND reforms are professionals, as they are in the front-line of service provision. The new SEND policy framework brings about a number of challenges and opportunities for these professionals on how to better meet the needs of children and young people with SEND, but they also require substantial adjustments to their roles and professional realm of action. Although professionals seem to agree in principle and to a large extent with many of the changes introduced, several constraints and barriers identified 
by them suggest a rather fragmented implementation of the SEND policy to date. Differences between professional groups regarding the implementation of the SEND policy are also illustrative that actions are needed to adequately implement holistic service provision.

This study provides clear evidence that there is a hiatus between political ideology and service provision, which is preventing some theoretically sound principles (such as multiagency working and holistic service provision) from being smoothly and adequately implemented. Clear guidelines and systematic and standardised training seem to be needed on: transdisciplinary problem solving and role sharing for a more effective collaborative partnership between disciplines; on universal participation-focused child assessment and classification procedures for a holistic evidence-based education health and care assessment procedure; continuous professional development within specialist professional groups in order to support the adjustment and extension of the boundaries of their professional realm of action in more standardised ways, which conform to specific professional standards but also to policy requirements. 


\section{References}

Atkinson, Cathy, Sandra Dunsmuir, Jane Lang, and Sarah Wright. 2015. "Developing a competency framework for the initial training of educational psychologists working with young people aged 16-25." Educational Psychology in Practice, 31 (2): 159-173.

Ball, S. J. 2015. "What is policy? 21 years later: reflections on the possibilities of policy research" Discourse: studies in the Cultural Politics of Education, 36 (3): 306-313.

Broach, S., L. Clements, and J. Read. 2016. Disabled Children: A Legal Handbook. 2nd ed. London: Legal.

Castro, S., \& Palikara, O. 2016. "Mind the Gap: The New Special Educational Needs and Disability Legislation in England." Frontiers in Education, 1 (4): 1-9.

Clarke, S., Sloper, P., Moran, N., Cusworth, L., Franklin, A., \& Beecham, J. 2011. "Multiagency transition services: greater collaboration needed to meet the priorities of young disabled people with complex needs as they move into adulthood." Journal of Integrated Care, 19 (5): 30-40.

Curran, H., Mortimore, T., \& Riddell, R. 2017. "Special Educational Needs and Disabilities reforms 2014: SENCos' perspectives of the first six months." British Journal of Special Education, 44 (1): 46-64.

Department for Education and Department of Health. 2015. Special Educational Needs and Disability Code of Practice: 0 to 25 years. London: HMSO.

Department for Education. 2011. Support and Aspiration: a new approach to special educational needs and disability - a consultation. London: DfE. doi: http:// media.education.gov.uk/assets/files/pdf/g/ green\%20paper\%20presentation.pdf.

Department for Education. 2014. Children and Families Act. London: DfE. 
Department for Education. 2015. The Special Educational Needs and Disability Pathfinder Programme Evaluation: Final Impact Research Report. London: DfE.

Dockrell, Julie Elizabeth, Peter Howell, Diane Leung, and Andrew JB Fugard. 2017.

"Children with speech language and communication needs in England: challenges for Practice." Frontiers in Education, 2: 35.

Eccles, A. 2012. "Partnerships: The politics of agendas and policy implementation. In The transformation of children's services: Examining and debating the complexities of inter/professional working”, edited by Forbes, J. and Watson, C. Abingdon: Routledge.

Friel, John, and Melinda Nettleton. 2015. Special Needs and Legal Entitlement: The Essential Guide to Getting out of the Maze. Jessica Kingsley Publishers.

Hellawell, B. 2017. "'There is Still a Long Way to Go to be Solidly Marvellous': Professional Identities, Performativity and Responsibilisation Arising From the Send Code of Practice 2015." British Journal of Educational Studies, 1-17.

Lamb, B. 2013. “Accountability, the local offer and SEND reform: A cultural revolution?” In SEN Policy Research Forum (2015) How will accountability work in the new SEND legislative system? Journal of Research in Special Educational Needs, 15 (1): 57-79.

Lindqvist, G., Nilholm, C., Almqvist, L., and Wetso, G.M. 2011. "Different agendas? The views of different occupational groups on special needs education.” European Journal of Special Needs Education, 26 (2): 143-157.

McKean, C., Law, J., Laing, K., Cockerill, M., Allon-Smith, J., McCartney, E., and Forbes, J. 2017. "A qualitative case study in the social capital of co-professional collaborative co-practice for children with speech, language and communication needs." International Journal of Language and Communication Disorders, 52 (4): 514-527. 
Milbourne, L., Macrae, S., and Maguire, M. 2003. "Collaborative solutions or new policy problems: exploring multi-agency partnerships in education and health work." Journal of Education Policy, 18 (1): 19-35.

Norwich, B. 2014. "Changing policy and legislation and its effects on inclusive and special education: a perspective from England.” British Journal of Special Education, 41 (4): $403-425$.

Norwich, B. 2016. “Conceptualising special educational needs using a bio-psycho-social model in England: the prospects and challenges of using the International Classification of Functioning framework." Frontiers in Education, 1 (5): 1-12.

Norwich, B., \& Eaton, A. 2015. "The new special educational needs (SEN) legislation in England and implications for services for children and young people with social, emotional and behavioural difficulties." Emotional and Behavioural Difficulties, 20 (2): 117-132.

Palikara, O., Lindsay, G., Cullen, M. A., \& Dockrell, J. 2007. "Working together? The practice of educational psychologists and speech and language therapists with children with specific speech and language difficulties." Educational and Child Psychology, 24: 7788.

Pearson, S., Mitchell, R., and Rapti, M. 2015. "I will be "fighting” even more for pupils with SEN': SENCOs' role predictions in the changing English Policy context.” Journal of Research in Special Educational Needs, 15 (1): 48-56. 